\title{
Implementation of Associated Hermite FDTD Method in Handling INBCs for Shielding Analysis
}

\author{
Lihua Shi, Zhengyu Huang, Qing Si, and Yinghui Zhou \\ National Key Laboratory on Electromagnetic Environmental Effects and Electro-optical Engineering, \\ PLA University of Science and Technology, Nanjing 210007, China
}

Correspondence should be addressed to Zhengyu Huang; huangzynj@163.com

Received 17 December 2015; Accepted 6 April 2016

Academic Editor: Rodolfo Araneo

Copyright (C) 2016 Lihua Shi et al. This is an open access article distributed under the Creative Commons Attribution License, which permits unrestricted use, distribution, and reproduction in any medium, provided the original work is properly cited.

For modeling of electrically thin conductive shields, the unconditionally stable Associated Hermite (AH) FDTD scheme is combined with the impedance network boundary conditions (INBCs) in this paper. The two-port network equations of INBCs in frequency domain are transformed into $\mathrm{AH}$ domain to represent the relationship of tangential components of the electric and magnetic fields at faces of the shield. The established AH-INBCs shielding boundaries are incorporated into a set of implicit equations to calculate the expansion coefficients vectors of electromagnetic fields in the computational domain. The method is free of CFL condition and no convolution integral operation for solving the conventional INBCs-FDTD is involved. Numerical example shows that, compared with analytical solutions and conventional FDTD method, the proposed algorithm is efficient and accurate.

\section{Introduction}

The finite-difference time-domain method $[1,2]$ has been widely used for many transient electromagnetic (EM) problems. In modeling of the electrically thin conductive layers (TCLs) by FDTD method $[3,4]$, it is needed to use sufficiently fine spatial grid size to achieve good accuracy and very small time step is required to meet the well-known CourantFriedrich-Levy (CFL) stability condition [2]. To solve this problem, many approaches such as spatial filtering [5], subgridding technologies [6,7], and subcell methods [8-14] have been developed. One of efficient subcell methods is based on the impedance network boundary conditions (INBCs) [10] and developed in recent years [11-16]. This method allows the shield region to be eliminated from the FDTD computational domain and to be reduced to a sheet of INBCs shielding boundary to model the coupling EM field tangential components on the shield surfaces. The self- and mutual impendences are used to represent the relationship of field components on both sides of the thin layer in frequency domain analytically. The time-domain transient expressions can be obtained by using inverse Fourier transform and recursive convolution approaches [4, 9-13]. The recursive process can be achieved by establishing a parametric model of the transient impedances, such as using a series of rational functions with vector fitting method [17], or incorporated with delay extraction techniques $[18,19]$ to reduce the complexity of the rational model to approximate the frequencydependent impedances. Under these cases, the FDTD methods need to be modified to handle frequency-dependent parameters and it is still required to meet the CFL stability condition.

In this paper, the unconditionally stable Associated Hermite (AH) FDTD proposed recently [20] is incorporated with INBC two-port network equations to analyze the shielding problems. The main idea for AH-FDTD method is to use $\mathrm{AH}$ orthogonal functions as temporal basis and testing functions to expand Maxwell's equations. By using Galerkin's principle, the time variable is eliminated from the calculation, and the time step is not limited by CFL stability condition. Incorporated with this unconditionally stable method, INBCs technologies are rendered much more efficient than previous method. Frequency-domain impendences can be directly transformed into $\mathrm{AH}$ transformation matrixes [21]. By using the unique isomorphism of the $\mathrm{AH}$ function with its Fourier transform [22, 23], the INBCs boundary equations 
are then realized by multiplying an $\mathrm{AH}$ transformation matrix. A set of implicit equations incorporated with the $\mathrm{AH}-$ INBCs equations is derived to calculate the electromagnetic field expanding coefficients. The time-domain waveform or frequency-dependent results such as shielding effectiveness can be reconstructed directly from these expanding coefficients.

This paper is organized as follows. In Section 2, the INBCs in AH domain (AH-INBCs) are derived by using the $\mathrm{AH}$ transformation matrix and the unconditionally stable scheme based on the AH-INBCs (AH-INBCs-FDTD) is implemented in two-dimensional (2D) code. In Section 3, we adopted the same case as in [9] with the field penetration into thin multilayered conductive shells to calculate the shielding effectiveness and a comparison of our numerical results with the conventional FDTD method and the analytical solution is carried out. Finally, some conclusions are given in Section 4.

\section{Formulation of INBC in AH Domain}

2.1. AH Transformation Matrix for Frequency-Dependent Function. Given an input signal $x(t)$ and output signal $y(t)=$ $h(t) \otimes x(t)$, the time-domain convolution integral $\otimes$ can be represented by an $Q$-dimensional $A H$ transformation matrix $T=\left[t_{i, j}\right]_{1 \leq i, j \leq \mathrm{Q}}[21]$, where $t_{i, j}=\left\langle\phi_{j}(\widetilde{t}) \otimes h(t), \phi_{i}(\widetilde{t})\right\rangle$ and $\phi_{q}(\widetilde{t})$ $(q=i$ or $j$ ) is the $q$ th Associated Hermite (AH) functions [18]. $\widetilde{t}=\left(t-t_{f}\right) / \lambda$ is transformed time variables, where $t_{f}$ is a time-translating parameter and $\lambda$ is a time-scaling parameter. And then we can obtain the relationship between $x$ and $y$ in $\mathrm{AH}$ domain as follows:

$$
[y]=T[x]
$$

where the Q-tuple representation $[x]=\left[\begin{array}{lll}x_{0} & \cdots & x_{Q-1}\end{array}\right]^{T}$ and $[y]=\left[\begin{array}{lll}y_{0} & \cdots & y_{\mathrm{Q}-1}\end{array}\right]^{T}$, which both consisted of $\mathrm{AH}$ expanding coefficients. One can also calculate $T$ from the frequency-domain data by using isomorphism property of $\mathrm{AH}$ function [21]. For a proper approximation with $Q$ $\mathrm{AH}$ expansion coefficients, the parameters selection can be selected according to $[20,24]$ :

$$
\begin{aligned}
& Q \approx \frac{1.7\left(\sqrt{t_{\mathrm{Q}} w_{\mathrm{Q}} \pi}-1.8\right)^{2}}{\pi}, \\
& \lambda \approx \frac{t_{\mathrm{Q}}}{2(\sqrt{\pi \mathrm{Q} / 1.7}+1.8)},
\end{aligned}
$$

where $t_{\mathrm{Q}}$ and $w_{\mathrm{Q}}$ are the time and frequency support of the $\mathrm{AH}$ basis $\left\{\phi_{0} \cdots \phi_{\mathrm{Q}-1}\right\}$, respectively. One should note that the bigger $Q$ could achieve more accuracy as well as more memory storage. Therefore, (2) means the minimum number of $\mathrm{AH}$ functions for a given time and frequency support.

Here, we give an example to illustrate the effectiveness of the $\mathrm{AH}$ transformation matrix for reconstructing the output response from an input signal as shown in Figure 1. Given the time and frequency support $t_{\mathrm{Q}}=4 \mathrm{~s}$ and $w_{\mathrm{Q}}=12 \mathrm{~Hz}$, the parameters for $\mathrm{AH}$ basis can be obtained as $\lambda=0.16$ and $Q=$ 60 from formulations (2)-(3), and time-translating parameter $t_{f}$ is set as $0.5 t_{Q}$ to guarantee the causal responses. Figure 1(a) shows an input signal, which is a Gaussian-differential pulse. Figure 1(b) is the AH functions with the parameters selected above. The $Q$-tuple representation $[x]$ can be calculated based on these functions. If the frequency-dependent system function is $H(\omega)=(j \omega+205) /(j \omega+5)^{2}$, then its $\mathrm{AH}$ transformation matrix $T$ can be calculated. Figure $1(c)$ shows the absolute value for $T$. Finally, the output $y(t)$ can be reconstructed from $Q$-tuple representation $[y]$, which can be calculated from (1). The reconstructed waveform fits well with the result from IFFT method as shown in Figure 1(d).

2.2. INBCs in AH Domain. We now consider a simple TCL region $\Omega_{s}$ of thickness $d$, relative permittivity $\varepsilon_{s}$, relative permeability $\mu_{s}$, and conductivity $\sigma_{s}$ as shown in Figure 2. It is illuminated by a TEz plane wave in the air region $\Omega_{0}$ from surface $\Gamma_{l}$ to $\Gamma_{r}$. It is assumed that (1) the thin layer is modeled as a homogeneous medium, characterized by a constant effective conductivity, permittivity, and permeability and (2) the propagation constants within the thin layer are much greater than the respective quantity in the surrounding materials [9]. Then, the frequency-domain tangential electric fields $E_{y l}$ and $E_{y r}$ and magnetic fields $H_{z l}$ and $H_{z r}$ satisfy the INBC two-port network equations [12] described as follows:

$$
\begin{aligned}
& E_{y l}(\omega)=Z_{l}(\omega) H_{z l}(\omega)-Z_{t}(\omega) H_{z r}(\omega), \\
& E_{y r}(\omega)=-Z_{r}(\omega) H_{z r}(\omega)+Z_{t}(\omega) H_{z l}(\omega),
\end{aligned}
$$

where $Z_{l}$ and $Z_{r}$ are the self-impedances of faces $\Gamma_{l}$ and $\Gamma_{r}$, respectively, and $Z_{t}$ is the mutual impedance between faces $\Gamma_{l}$ and $\Gamma_{r}$. For a homogeneous layer, the impedances are given by $Z_{l}(\omega)=Z_{r}(\omega)=\eta \operatorname{coth}(\gamma d)$ and $Z_{t}(\omega)=\eta / \sinh (\gamma d)$, where $\eta=\sqrt{\mu_{s} /\left(\varepsilon_{s}+\sigma_{s} / j \omega\right)}$ and $\gamma=j \omega \sqrt{\mu_{s}\left(\varepsilon_{s}+\sigma_{s} / j \omega\right)}$ are the intrinsic impedance and the propagation constant inside the layer, respectively. For the case of multilayer, the impedance representations can also be calculated by transmission matrix of the multilayered slab [9].

According to the previous analysis of $\mathrm{AH}$ transformation matrix in Section 2.1, (4) of the two-port network in frequency domain can be transformed into the Q-tuple representations as

$$
\begin{aligned}
& {\left[E_{y l}\right]=T_{z l}\left[H_{z l}\right]-T_{z t}\left[H_{z r}\right],} \\
& {\left[E_{y r}\right]=-T_{z r}\left[H_{z r}\right]+T_{z t}\left[H_{z l}\right],}
\end{aligned}
$$

where $\left[E_{y l}\right],\left[E_{y r}\right],\left[H_{z l}\right]$, and $\left[H_{z r}\right]$ are the Q-tuple electric and magnetic field tangential components. $T_{z l}, T_{z r}$, and $T_{z t}$ are the $\mathrm{Q}$-dimension $\mathrm{AH}$ transform matrixes to the frequency-dependent self- and mutual transfer impedances $Z_{l}, Z_{r}$, and $Z_{t}$, respectively.

2.3. The INBCs Implementation for $A H$-FDTD. In the region $\Omega_{0}$, the 2D time-domain Maxwell's equations for TEz case can 


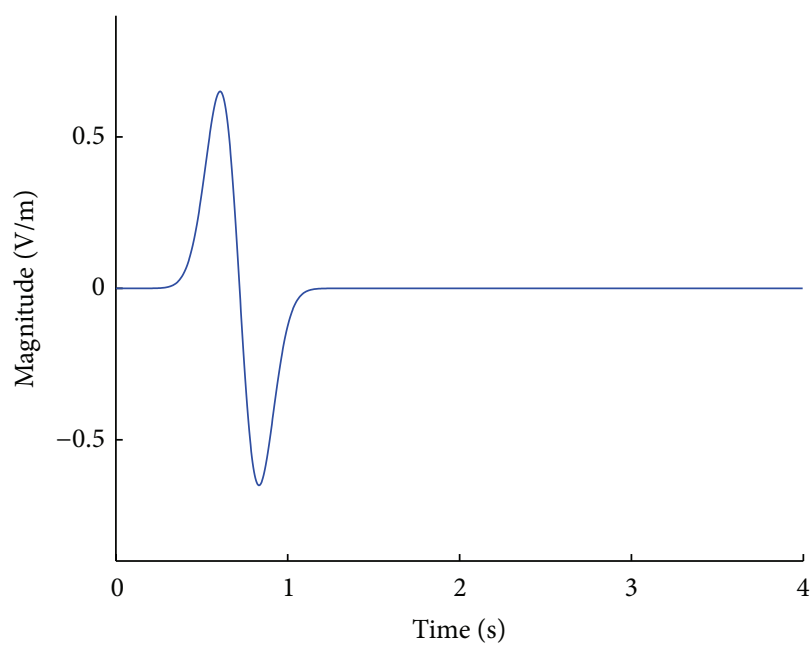

(a)

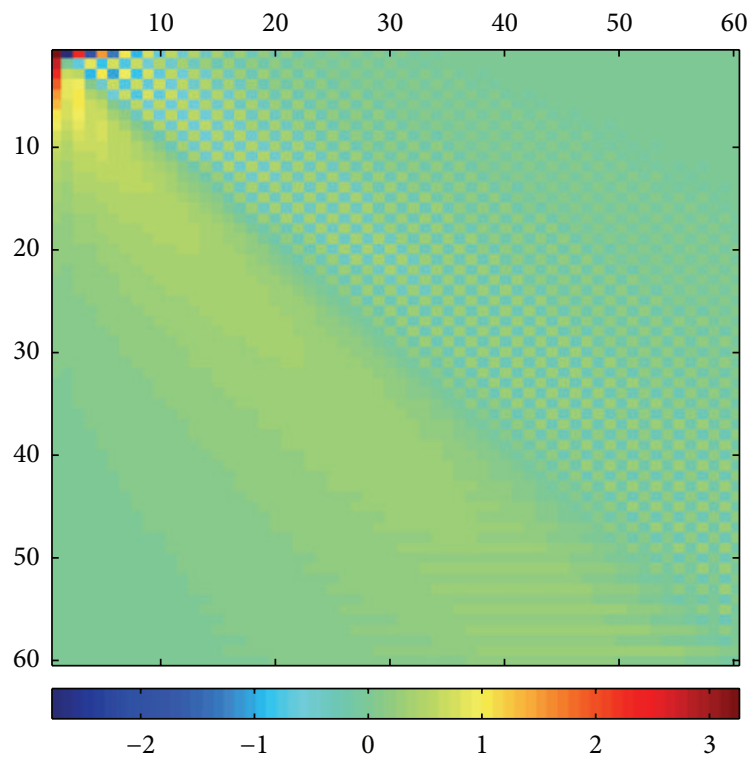

(c)

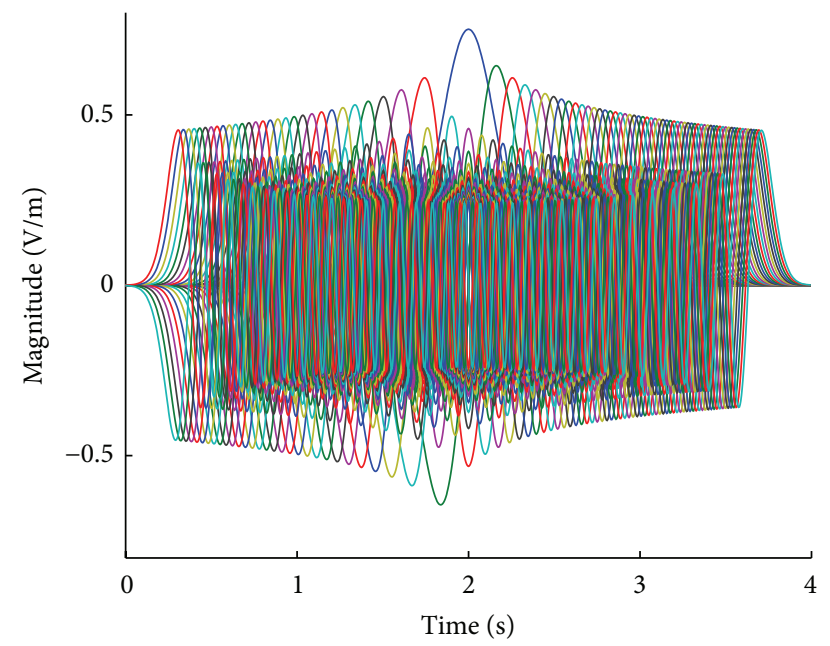

(b)

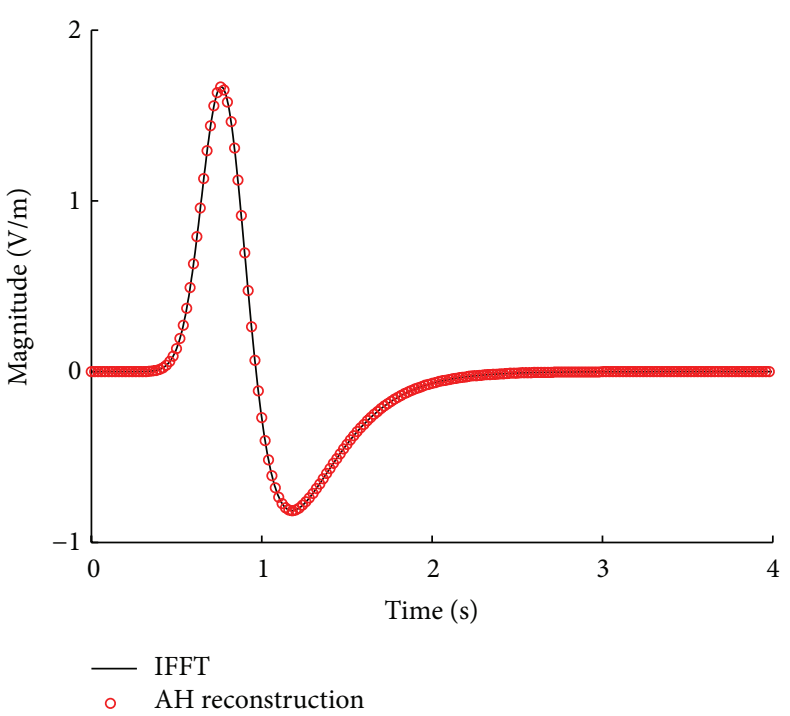

(d)

FIgURE 1: AH transformation for calculating an output signal from an input signal. (a) An input signal. (b) The AH basis with the parameters: $\lambda=0.16, Q=60$, and $t_{f}=0.5 t_{\mathrm{Q}}$. (c) The absolute value of the transformation matrix for $H(\omega)$. (d) The output signals $y$ calculated by AH reconstruction method and IFFT.

be expanded by AH basis $\left\{\phi_{0} \cdots \phi_{\mathrm{Q}-1}\right\}$ and converted into the $Q$-tuple expansion coefficients representation [20] as

$$
\begin{aligned}
& {[\alpha]\left[E_{x}\right]_{i, j}=\left.\bar{C}_{y}^{E}\right|_{i, j}[\beta]\left(\left[H_{z}\right]_{i, j}-\left[H_{z}\right]_{i, j-1}\right)} \\
& -\frac{\lambda[\beta]\left[J_{x}\right]_{i, j}}{\varepsilon_{0}}+\left[E_{x}^{t_{0}}\right]_{i, j}, \\
& {[\alpha]\left[E_{y}\right]_{i, j}=-\left.\bar{C}_{x}^{E}\right|_{i, j}[\beta]\left(\left[H_{z}\right]_{i, j}-\left[H_{z}\right]_{i-1, j}\right)} \\
& \quad-\frac{\lambda[\beta]\left[J_{y}\right]_{i, j}}{\varepsilon_{0}}+\left[E_{y}^{t_{0}}\right]_{i, j}, \\
& {[\alpha]\left[H_{z}\right]_{i, j}=\left.\bar{C}_{y}^{H}\right|_{i, j}[\beta]\left(\left[E_{x}\right]_{i, j+1}-\left[E_{x}\right]_{i, j}\right)-\left.\bar{C}_{x}^{H}\right|_{i, j}[\beta]} \\
& \quad \cdot\left(\left[E_{y}\right]_{i+1, j}-\left[E_{y}\right]_{i, j}\right)+\left[H_{z}^{t_{0}}\right]_{i, j},
\end{aligned}
$$

where

$$
\begin{aligned}
& {[\alpha]=\left[\begin{array}{ccccc}
-\sqrt{\frac{1}{2}} & 1 & & & \\
& & \ddots & & \\
& -\sqrt{\frac{(Q-2)}{(Q-1)}} & & 1 & \\
\phi_{0}\left(\widetilde{t_{0}}\right) & \phi_{1}\left(\widetilde{t_{0}}\right) & \cdots & \phi_{Q-1}\left(\widetilde{t_{0}}\right)
\end{array}\right],} \\
& {[\beta]=\left[\begin{array}{lllll}
\sqrt{2} & & & & \\
& \sqrt{1} & & & \\
& \ddots & & \\
& & \sqrt{\frac{2}{(Q-1)}} &
\end{array}\right]}
\end{aligned}
$$




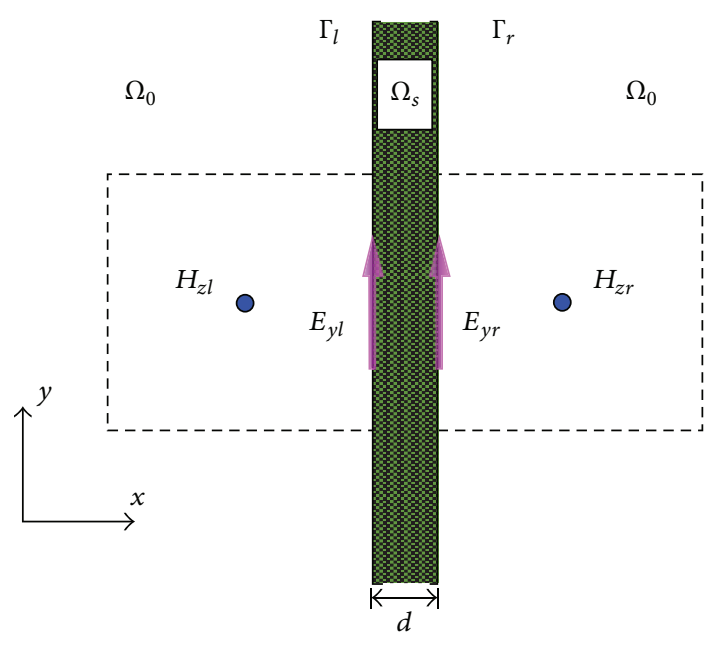

FIgURE 2: Configuration of a TCL illuminated by a TEz plane wave.

$$
\begin{aligned}
& \left.\bar{C}_{x}^{E}\right|_{i, j}=\frac{\lambda}{\varepsilon_{0} \Delta \bar{x}_{i}}, \\
& \left.\bar{C}_{y}^{E}\right|_{i, j}=\frac{\lambda}{\varepsilon_{0} \Delta \bar{y}_{j}}, \\
& \left.\bar{C}_{x}^{H}\right|_{i, j}=\frac{\lambda}{\mu_{0} \Delta x_{i}}, \\
& \left.\bar{C}_{y}^{H}\right|_{i, j}=\frac{\lambda}{\mu_{0} \Delta y_{j}}, \\
& {\left[E_{\xi}^{t_{0}}\right]_{i, j}=\left[\begin{array}{llll}
0 & \cdots & 0 & E_{\xi}\left(i, j, t_{0}\right)
\end{array}\right]^{T},} \\
& {\left[H_{z}^{t_{0}}\right]_{i, j}=\left[\begin{array}{llll}
0 & \cdots & 0 & H_{z}\left(i, j, t_{0}\right)
\end{array}\right]^{T} .}
\end{aligned}
$$

$\left[E_{\xi}\right]_{i, j}(\xi=x, y)$ and $\left[H_{z}\right]_{i, j}$ are Q-tuple components for electric and magnetic fields. $E_{\xi}\left(i, j, t_{0}\right)$ and $H_{z}\left(i, j, t_{0}\right)$ are initial conditions. One can find the more detailed derivation in Appendix.

To eliminate the electric field, we apply (6)-(7) to (8):

$$
\begin{gathered}
b_{l}\left[H_{z}\right]_{i-1, j}+b_{r}\left[H_{z}\right]_{i+1, j}+a\left[H_{z}\right]_{i, j}+b_{u}\left[H_{z}\right]_{i, j+1} \\
+b_{d}\left[H_{z}\right]_{i, j-1}=[J]_{i, j},
\end{gathered}
$$

where

$$
\begin{aligned}
& b_{l}=\left.\left.\bar{C}_{x}^{E}\right|_{i, j} \bar{C}_{x}^{H}\right|_{i, j}[\beta][\alpha]^{-1}[\beta], \\
& b_{r}=\left.\left.\bar{C}_{x}^{E}\right|_{i+1, j} \bar{C}_{x}^{H}\right|_{i, j}[\beta][\alpha]^{-1}[\beta], \\
& b_{u}=\left.\left.\bar{C}_{y}^{E}\right|_{i, j+1} \bar{C}_{y}^{H}\right|_{i, j}[\beta][\alpha]^{-1}[\beta], \\
& b_{d}=\left.\left.\bar{C}_{y}^{E}\right|_{i, j} \bar{C}_{y}^{H}\right|_{i, j}[\beta][\alpha]^{-1}[\beta], \\
& a=-\left(b_{u}+b_{d}+b_{l}+b_{r}+[\alpha]\right),
\end{aligned}
$$

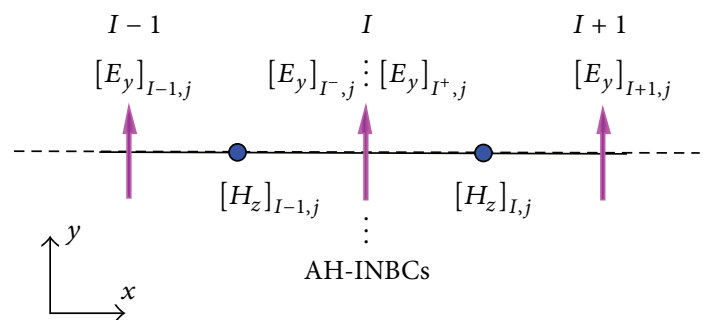

Figure 3: AH-FDTD grid for the INBC implementation.

$$
\begin{aligned}
{[J]_{i j}=} & \frac{\left.\bar{C}_{y}^{H}\right|_{i, j} \lambda[\beta][\alpha]^{-1}[\beta]\left(\left[J_{x}\right]_{i, j+1}-\left[J_{x}\right]_{i, j}\right)}{\varepsilon_{0}} \\
& -\frac{\left.\bar{C}_{x}^{H}\right|_{i, j} \lambda[\beta][\alpha]^{-1}[\beta]\left(\left[J_{y}\right]_{i+1, j}-\left[J_{y}\right]_{i, j}\right)}{\varepsilon_{0}} \\
& -\left.\bar{C}_{y}^{H}\right|_{i, j}[\beta][\alpha]^{-1}\left(\left[P_{x}^{t_{0}}\right]_{i, j+1}-\left[P_{x}^{t_{0}}\right]_{i, j}\right) \\
& +\left.\bar{C}_{x}^{H}\right|_{i, j}[\beta][\alpha]^{-1}\left(\left[P_{y}^{t_{0}}\right]_{i+1, j}-\left[P_{y}^{t_{0}}\right]_{i, j}\right) \\
& -\left[H_{z}^{t_{0}}\right]_{i, j} .
\end{aligned}
$$

Equation (10) is related to a five-diagonal banded coefficient matrix for the AH-FDTD formulations in region $\Omega_{0}$. Its solving method using the lower-upper (LU) decomposition has been discussed in [20]. Here, we continue to develop the AH-FDTD method to INBCs boundary condition.

The shield region $\Omega_{s}$ in the $y-z$ plane is eliminated from the computational domain and replaced by the AH-INBCs at point $(i=I)$ as shown in Figure 3. The magnetic field on the shield surfaces, $\left[H_{z}\right]_{I^{-}, j}$ and $\left[H_{z}\right]_{I^{+}, j}$, is approximated as $\left[H_{z}\right]_{I^{-}, j} \approx\left[H_{z}\right]_{I-1, j}$ and $\left[H_{z}\right]_{I^{+}, j} \approx\left[H_{z}\right]_{I, j}$ and the $y$ components of the electric field, $\left[E_{y}\right]_{I^{-}, j}$ and $\left[E_{y}\right]_{I^{+}, j}$, can be rewritten from the coupling equations (5) as

$$
\begin{aligned}
& {\left[E_{y}\right]_{I^{-}, j}=T_{z l}\left[H_{z}\right]_{I-1, j}-T_{z t}\left[H_{z}\right]_{I, j},} \\
& {\left[E_{y}\right]_{I^{+}, j}=-T_{z r}\left[H_{z}\right]_{I, j}+T_{z t}\left[H_{z}\right]_{I-1, j} .}
\end{aligned}
$$

Applying (12) into (7) and combining (6)-(8) at point ( $i=$ $I-1)$ for the magnetic field $\left[H_{z}\right]_{I-1, j}$, we can also eliminate the electric field and obtain a modified equation for (10). Most coefficients in (10) remain the same as before except $b_{r}$ and $a$, which are slightly changed as follows:

$$
\begin{aligned}
& b_{r}=\left.\bar{C}_{x}^{H}\right|_{I-1, j}[\beta] T_{z t}, \\
& a=-\left(b_{u}+b_{d}+b_{l}+\left.\bar{C}_{x}^{H}\right|_{I-1, j}[\beta] T_{z l}+[\alpha]\right) .
\end{aligned}
$$


TABLE 1: Composition of multilayered panels [9].

\begin{tabular}{|c|c|c|c|c|}
\hline Panel & Number of layers & Layer conductivity $(\mathrm{S} / \mathrm{m})$ & Layer relative permittivity & Layer thickness (mm) \\
\hline $\mathrm{A}$ & 1 & $\sigma_{1}=10^{4}$ & $\varepsilon_{r 1}=2$ & $d_{1}=1$ \\
\hline \multirow{3}{*}{ B } & \multirow{3}{*}{3} & $\sigma_{1}=10^{4}$ & $\varepsilon_{r 1}=2$ & $d_{1}=0.6$ \\
\hline & & $\sigma_{2}=50$ & $\varepsilon_{r 2}=4$ & $d_{2}=0.6$ \\
\hline & & $\sigma_{3}=10^{3}$ & $\varepsilon_{r 3}=3$ & $d_{3}=0.6$ \\
\hline \multirow{3}{*}{$\mathrm{C}$} & \multirow{3}{*}{5} & $\sigma_{1}=\sigma_{3}=10^{4}$ & $\varepsilon_{r 1}=\varepsilon_{r 3}=2$ & $d_{1}=d_{3}=0.2$ \\
\hline & & $\sigma_{2}=\sigma_{4}=50$ & $\varepsilon_{r 2}=\varepsilon_{r 4}=4$ & $d_{2}=d_{4}=0.2$ \\
\hline & & $\sigma_{5}=10^{3}$ & $\varepsilon_{r 5}=3$ & $d_{5}=0.2$ \\
\hline \multirow{3}{*}{$\mathrm{D}$} & \multirow{3}{*}{9} & $\sigma_{1}=\sigma_{3}=\sigma_{8}=10^{4}$ & $\varepsilon_{r 1}=\varepsilon_{r 3}=\varepsilon_{r 8}=2$ & $d_{1}=d_{3}=d_{8}=0.2$ \\
\hline & & $\sigma_{2}=\sigma_{4}=\sigma_{6}=50$ & $\varepsilon_{r 2}=\varepsilon_{r 4}=\varepsilon_{r 6}=4$ & $d_{2}=d_{4}=d_{6}=0.2$ \\
\hline & & $\sigma_{5}=\sigma_{7}=\sigma_{9}=10^{3}$ & $\varepsilon_{r 5}=\varepsilon_{r 7}=\varepsilon_{r 9}=3$ & $d_{5}=d_{7}=d_{9}=0.2$ \\
\hline
\end{tabular}

Similarly, at point $(i=I)$ for $\left[H_{z}\right]_{I, j}$, we can obtain the modified coefficients as follows:

$$
\begin{aligned}
& b_{l}=\left.\bar{C}_{x}^{H}\right|_{I, j}[\beta] T_{z t} \\
& a=-\left(b_{u}+b_{d}+b_{r}+\left.\bar{C}_{x}^{H}\right|_{I, j}[\beta] T_{z r}+[\alpha]\right) .
\end{aligned}
$$

By solving the modified (10), the whole unknown magnetic fields coefficients can be obtained, and by substituting the magnetic field into (6) and (7), the electric field coefficients can also be obtained. Then, the time-domain fields could be reconstructed from these coefficients. Mur's firstorder ABC in AH domain [20] can also be applied here to truncate the computation domain.

Furthermore, by using the unique property of isomorphism of the AH function in [21], the shielding effectiveness

$$
\mathrm{SE}=20 \log \left[\frac{\left|E_{\text {inc }}(f)\right|}{\left|E_{\text {tran }}(f)\right|}\right]
$$

can be reconstructed from these expansion coefficients directly. For example, $E_{\operatorname{tran}}(f)$ in SE can be reconstructed as

$$
E_{\text {tran }}(f)=\sum_{q=0}^{Q-1}(-j)^{q} E_{\text {tran }}^{q} \phi_{q}(2 \pi f),
$$

where $E_{\text {tran }}^{q}$ is the AH expansion coefficients.

\section{Numerical Results}

To verify the validity of the proposed method, the numerical examples in [9] for calculating the shielding effectiveness of multilayered composite panels are adopted. The four kinds of configurations A, B, C, and D are listed in Table 1.

In this example, a broad spectrum ranged from $100 \mathrm{~Hz}$ to $1 \mathrm{GHz}$ for analyzing the shielding effectiveness is investigated. For the proposed AH-INBCs-FDTD, the space interval is chosen as $\Delta x=c /\left(30 f_{\max }\right)=0.01 \mathrm{~m}$, and the total number size of computational cells is 120 . The time step, only used to accurately evaluate $Q$-tuple vector quantities $\left[J_{y}\right]_{i, j}$, is not involved in the AH-domain calculation. To compare with the conventional FDTD method, we can also set it as the following representation: $\Delta t=\operatorname{CFLN}(\Delta x / c)$, where CFLN is the CFL number. The first-order dispersive boundary condition $[20,25]$ is adopted as absorbing boundary conditions for the truncation of computation domain.

A Gaussian pulse plane wave described by

$$
E_{i}(t)=E_{0} \exp \left(-\frac{\left(t-t_{0}\right)^{2}}{\tau^{2}}\right) \mathrm{V} / \mathrm{m}
$$

with $\tau=15 \Delta x / c$ and $t_{0}=6 \tau$ is chosen as field excitations illuminating the panels. The total time and frequency support are set as $18 \mathrm{~ns}$ and $4.8 \mathrm{GHz}$. According to (2)-(3), the $\mathrm{AH}$ basis parameters are selected as $\lambda=5.42 \times 10^{-10}, Q=118$, and $t_{f}=9 \mathrm{~ns}$. And CFLN is selected as 1 for this example.

Then, the self- and mutual transfer impedances $Z_{l}, Z_{r}$, and $Z_{t}$ calculated by using the four configurations in Table 1 are projected into AH transformation matrixes $T_{z l}, T_{z r}$, and $T_{z t}$, respectively. For case $\mathrm{A}, T_{z l}$ is calculated the same as $T_{z r}$ and its absolute values of the matrix with $T_{z t}$ are plotted in Figure 4, respectively.

The shielding effectiveness is calculated by the proposed AH-INBCs-FDTD method and the analytical solution [26], respectively, for comparison. For the proposed method, the shielding effectiveness is calculated using (16)-(17) by specifying a series of sampling frequencies. The comparison results are shown in Figure 5. It demonstrates a good agreement between the two results.

To further validate the accuracy and efficiency for this unconditionally stable method, it is compared with the conventional FDTD by discretizing the panel with fine grids. Taking configuration A, for example, a single-layer panel with the thickness of $d_{1}=1 \mathrm{~mm}$ is analyzed with different cell sizes. For the conventional FDTD, CFLN for three cases remain the same as CFLN $=1$, while, for the proposed method, $\Delta t$ for four cases remain the same as $10 d_{1} / c$, and the equivalent CFLN are $1,10,50$, and 500, respectively.

The percentage errors of shielding effectiveness compared with analytical result are calculated as shown in Figure 6. The percentage error is defined as

$$
R=\frac{100\left|\mathrm{SE}_{\mathrm{AH}}(f)-\mathrm{SE}_{\text {Analytical }}(f)\right|}{\left|\mathrm{SE}_{\text {Analytical }}(f)\right|}
$$




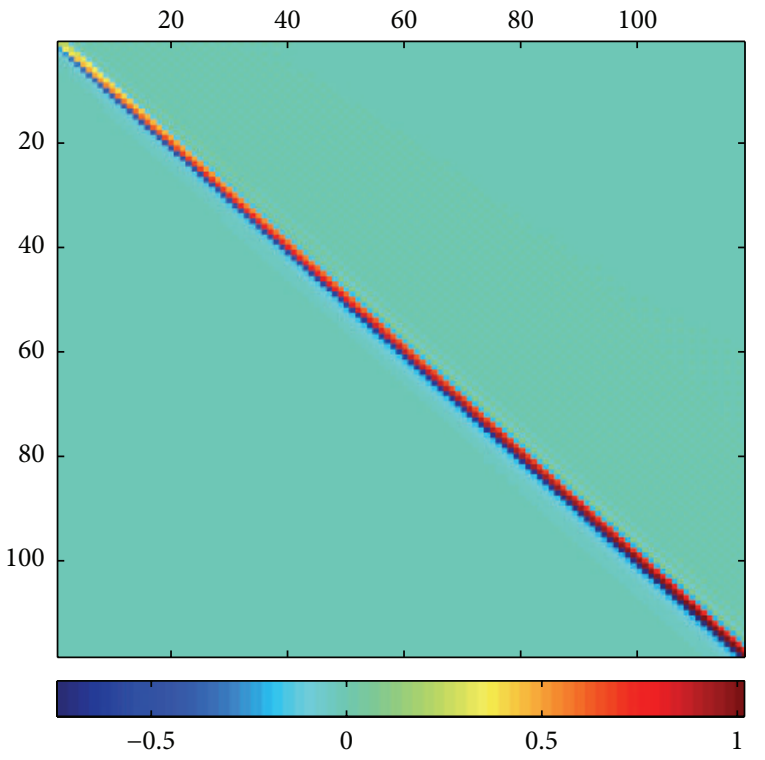

(a)

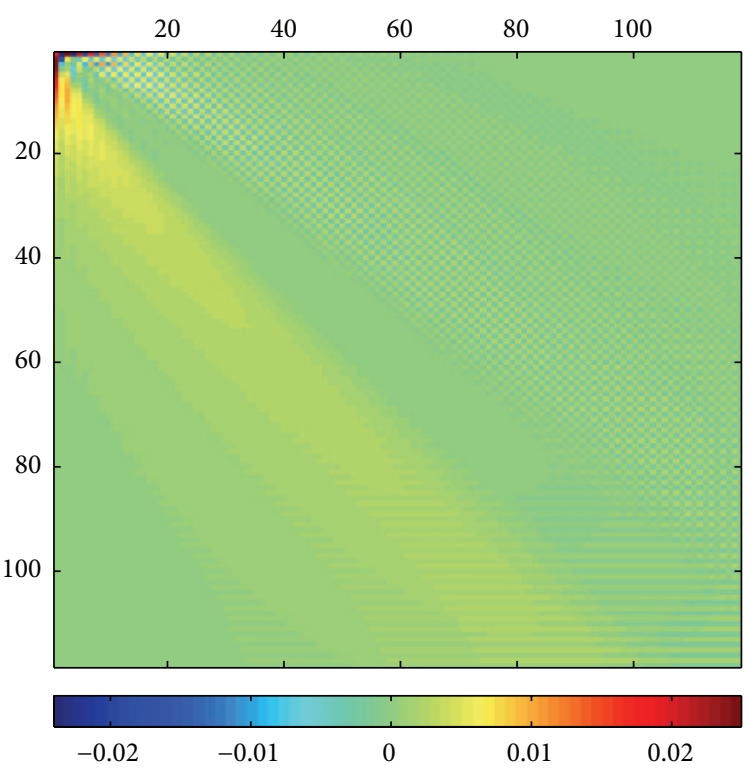

(b)

FIgUre 4: The absolute value of the transformation matrix for (a) $T_{z l}$ and (b) $T_{z t}$.

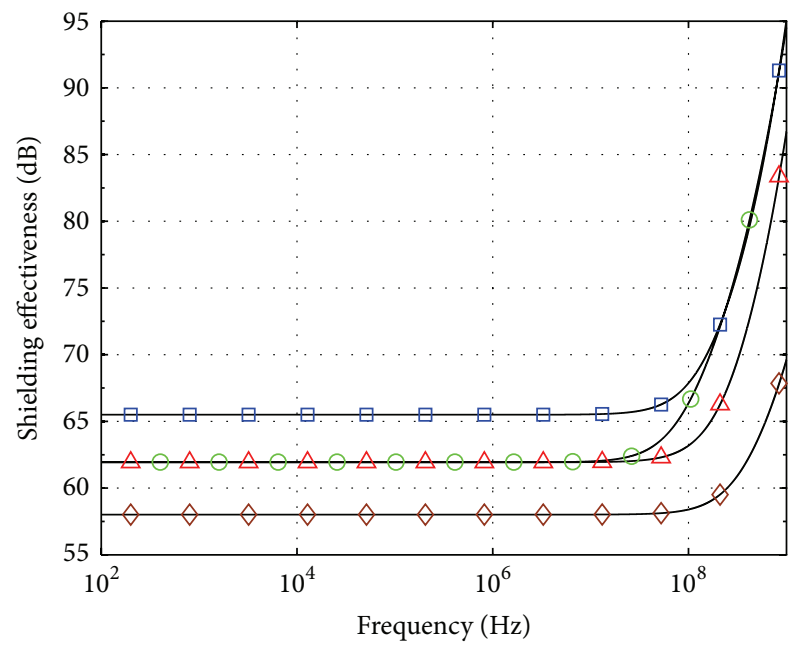

FIGURE 5: Comparison of shielding effectiveness of the panels in Table 1 by using the proposed method, A $(\square), B(\triangle), C(\diamond)$, and D (O) and the analytical method (-) [23].

Figure 6 shows that the percentage errors decrease with the increment of the number of the discretized cells for the conventional FDTD, while for the proposed method the results almost keep the same, with errors all below $0.001 \%$. Figure 7 shows an influence of different order $Q$ on the accuracy for the proposed method. It shows that the accuracy is improved with the increasing of $Q$ when keeping $\Delta x=d_{1}$ while the improvement is decreasing when $Q$ is bigger than 118. The maximum total simulation CPU time for conventional FDTD is $52.1 \mathrm{~s}$ for the case of $\Delta x=0.02 d_{1}$, while for the proposed method it is reduced to $2.6 \mathrm{~s}$.

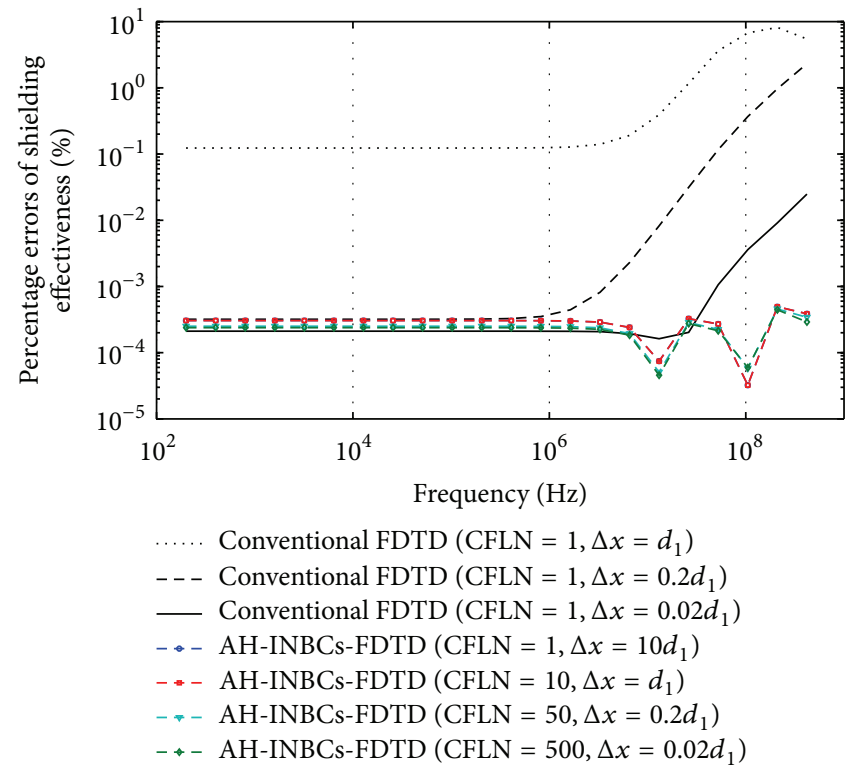

FIGURE 6: The percentage error of shielding effectiveness compared with analytical solution for the proposed method and conventional FDTD method under different size of space cell $\Delta x$.

\section{Conclusion}

An AH transformation matrix formulation for INBCs is presented for application in AH-FDTD. Compared to the conventional INBCs-FDTD method, this new approach does not need convolution or vector fitting to model the frequencydependent INBCs in time domain. Alternatively, it uses the $\mathrm{AH}$ transformation matrix to represent the frequencydependent INBCs and frequency-domain results can be 


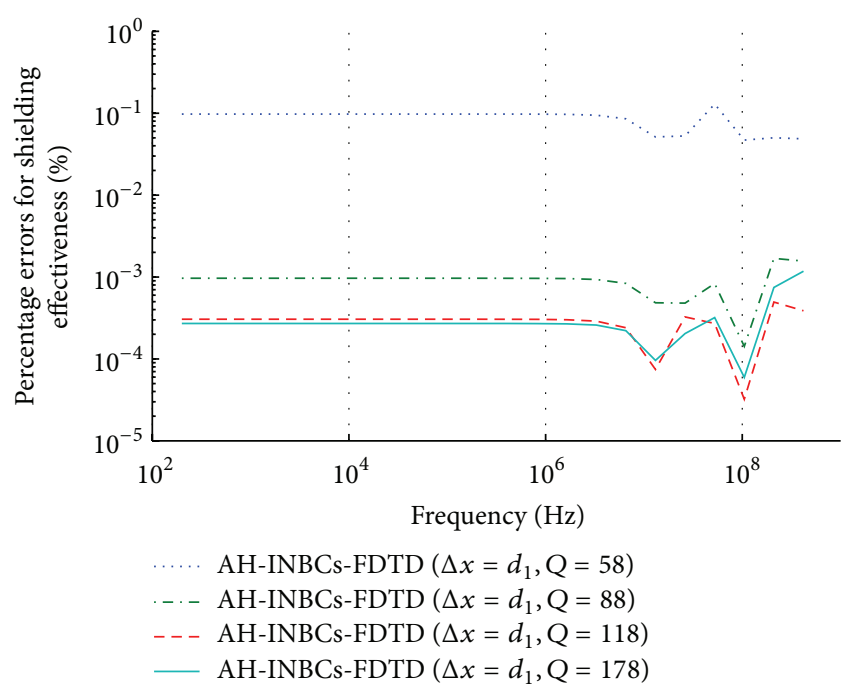

FIGURE 7: Influence of different order $Q$ affecting the accuracy.

directly reconstructed using the unique time-frequency isomorphism feature of $\mathrm{AH}$ basis. The proposed method has advantages over conventional FDTD in saving CPU time and avoiding the CFL constrains. The numerical accuracy can also be guaranteed for the relative larger CFLN. Although the proposed formulation is presented in $2 \mathrm{D}$ case, it can be combined with 3D AH-FDTD formulation to deal with threedimensional problems in future.

\section{Appendix}

Here, we give a brief derivation for (6)-(8). With simple and lossy media, the 2D time-domain Maxwell's equations for TEz case are

$$
\begin{aligned}
\varepsilon_{0} \frac{\partial}{\partial t} E_{x}(r, t) & =\frac{\partial}{\partial y} H_{z}(r, t)-J_{x}(r, t), \\
\varepsilon_{0} \frac{\partial}{\partial t} E_{y}(r, t) & =-\frac{\partial}{\partial x} H_{z}(r, t)-J_{y}(r, t), \\
\mu_{0} \frac{\partial}{\partial t} H_{z}(r, t) & =\frac{\partial}{\partial y} E_{x}(r, t)-\frac{\partial}{\partial x} E_{y}(r, t) .
\end{aligned}
$$

Similar to the derivation of method from [20], we can use $\mathrm{AH}$ functions to perform a temporal Galerkin testing procedure, and then we have

$$
\begin{gathered}
\frac{1}{\lambda}\left(E_{x}^{q+1}(r) \sqrt{\frac{q+1}{2}}-E_{x}^{q-1}(r) \sqrt{\frac{q}{2}}\right) \\
=\frac{1}{\varepsilon(r)} \frac{\partial}{\partial y} H_{z}^{q}(r)-\frac{J_{x}^{q}(r)}{\varepsilon(r)},
\end{gathered}
$$

$$
\begin{aligned}
& \frac{1}{\lambda}\left(E_{y}^{q+1}(r) \sqrt{\frac{q+1}{2}}-E_{y}^{q-1}(r) \sqrt{\frac{q}{2}}\right) \\
& =-\frac{1}{\varepsilon(r)} \frac{\partial}{\partial x} H_{z}^{q}(r)-\frac{J_{y}^{q}(r)}{\varepsilon(r)}, \\
& \frac{1}{\lambda}\left(H_{z}^{q+1}(r) \sqrt{\frac{q+1}{2}}-H_{z}^{q-1}(r) \sqrt{\frac{q}{2}}\right) \\
& =\frac{1}{\mu(r)} \frac{\partial}{\partial y} E_{x}^{q}(r)-\frac{1}{\mu(r)} \frac{\partial}{\partial x} E_{y}^{q}(r) .
\end{aligned}
$$

Then, apply spatial discretization to (A.2):

$$
\begin{aligned}
& \left.E_{x}\right|_{i, j} ^{q+1}-\left.\sqrt{\frac{q}{q+1}} E_{x}\right|_{i, j} ^{q-1} \\
& =\sqrt{\frac{2}{q+1}} \frac{\lambda}{\varepsilon_{i, j} \Delta \bar{y}_{j}}\left(\left.H_{z}\right|_{i, j} ^{q}-\left.H_{z}\right|_{i, j-1} ^{q}\right) \\
& -\left.\sqrt{\frac{2}{q+1}} \frac{\lambda}{\varepsilon_{i, j}} J_{x}\right|_{i, j} ^{q}, \\
& \left.E_{y}\right|_{i, j} ^{q+1}-\left.\sqrt{\frac{q}{q+1}} E_{y}\right|_{i, j} ^{q-1} \\
& =-\sqrt{\frac{2}{q+1}} \frac{\lambda}{\varepsilon_{i, j} \Delta \bar{x}_{i}}\left(\left.H_{z}\right|_{i, j} ^{q}-\left.H_{z}\right|_{i-1, j} ^{q}\right) \\
& -\left.\sqrt{\frac{2}{q+1}} \frac{\lambda}{\varepsilon_{i, j}} J_{y}\right|_{i, j} ^{q}, \\
& \left.H_{z}\right|_{i, j} ^{q+1}-\left.\sqrt{\frac{q}{q+1}} H_{z}\right|_{i, j} ^{q-1} \\
& =\sqrt{\frac{2}{q+1}} \frac{\lambda}{\mu_{i, j} \Delta y_{j}}\left(\left.E_{x}\right|_{i, j+1} ^{q}-\left.E_{x}\right|_{i, j} ^{q}\right) \\
& -\sqrt{\frac{2}{q+1}} \frac{\lambda}{\mu_{i, j} \Delta x_{i}}\left(\left.E_{y}\right|_{i+1, j} ^{q}-\left.E_{y}\right|_{i, j} ^{q}\right) .
\end{aligned}
$$

Initial conditions of electromagnetic fields are introduced. For instance, the initial condition of magnetic field can be represented as

$$
H_{z}\left(i, j, t_{0}\right)=\left.\sum_{q=0}^{Q-1} H_{z}\right|_{i, j} ^{q} \bar{\phi}_{q}\left(\widetilde{t_{0}}\right) .
$$


Then, a set of implicit equations with $Q$-tuple vector variables including initial conditions can be assembled from (A.3) and (A.4) as

$$
\begin{aligned}
{[\alpha]\left[E_{x}\right]_{i, j}=} & \left.\bar{C}_{y}^{E}\right|_{i, j}[\beta]\left(\left[H_{z}\right]_{i, j}-\left[H_{z}\right]_{i, j-1}\right) \\
& -\frac{\lambda[\beta]\left[J_{x}\right]_{i, j}}{\varepsilon_{0}}+\left[E_{x}^{t_{0}}\right]_{i, j}, \\
{[\alpha]\left[E_{y}\right]_{i, j}=} & -\left.\bar{C}_{x}^{E}\right|_{i, j}[\beta]\left(\left[H_{z}\right]_{i, j}-\left[H_{z}\right]_{i-1, j}\right) \\
& -\left.\frac{\lambda[\beta]\left[J_{y}\right]_{i, j}}{[\alpha]\left[H_{z}\right]_{i, j}=} \bar{C}_{y}^{H}\right|_{i, j}[\beta]\left(\left[E_{y}^{t_{0}}\right]_{i, j},\right. \\
& -\left.\bar{C}_{x}^{H}\right|_{i, j}[\beta]\left(\left[E_{y}\right]_{i+1, j}-\left[E_{y}\right]_{i, j}\right) \\
& +\left[H_{z}^{t_{0}}\right]_{i, j} .
\end{aligned}
$$

Finally, one can find that (A.5)-(A.7) are the formulations (6)-(8), respectively.

\section{Competing Interests}

The authors declare that there are no competing interests regarding the publication of this paper.

\section{Acknowledgments}

The project is supported by the National Natural Science Foundation of China (Grant nos. 51477183, 51477182, and 51407198.)

\section{References}

[1] K. S. Yee, "Numerical solution of initial boundary value problems involving maxwell's equations in isotropic media," IEEE Transactions on Antennas and Propagation, vol. 14, no. 3, pp. 302-307, 1966.

[2] A. Taflove and S. C. Hagness, Computational Electrodynamics: The Finite-Difference Time-Domain Method, Artech House, Norwood, Mass, USA, 2nd edition, 2000.

[3] J. G. Maloney and G. S. Smith, "A comparison of methods for modeling electrically thin dielectric and conducting sheets in the finite-difference time-domain method," IEEE Transactions on Antennas and Propagation, vol. 41, no. 5, pp. 690-694, 1993.

[4] S. Van den Berghe, F. Olyslager, and D. De Zutter, "Accurate modeling of thin conducting layers in FDTD," IEEE Microwave and Guided Wave Letters, vol. 8, no. 2, pp. 75-77, 1998.

[5] C. Chang and C. D. Sarris, "A spatially filtered finite-difference time-domain scheme with controllable stability beyond the CFL limit: theory and applications," IEEE Transactions on Microwave Theory and Techniques, vol. 61, no. 1, pp. 351-359, 2013.

[6] S. S. Zivanovic, K. S. Yee, and K. K. Mei, "A subgridding method for the time-domain finite-difference method to solve Maxwell's equations," IEEE Transactions on Microwave Theory and Techniques, vol. 39, no. 3, pp. 471-479, 1991.
[7] K. Xiao, D. J. Pommerenke, and J. L. Drewniak, "A threedimensional FDTD subgridding algorithm with separated temporal and spatial interfaces and related stability analysis," IEEE Transactions on Antennas and Propagation, vol. 55, no. 7, pp. 1981-1990, 2007.

[8] J. G. Maloney and G. S. Smith, "The use of surface impedance concepts in the finite-difference time-domain method," IEEE Transactions on Antennas and Propagation, vol. 40, no. 1, pp. 3848, 1992.

[9] M. S. Sarto, "A new model for the FDTD analysis of the shielding performances of thin composite structures," IEEE Transactions on Electromagnetic Compatibility, vol. 41, no. 4, pp. 298-306, 1999.

[10] M. Feliziani and F. Maradei, "Fast computation of quasi-static magnetic fields around nonperfectly conductive shields," IEEE Transactions on Magnetics, vol. 34, no. 5, pp. 2795-2798, 1998.

[11] M. Feliziani, F. Maradei, and G. Tribellini, "Field analysis of penetrable conductive shields by the finite-difference timedomain method with Impedance Network Boundary Conditions (INBC's)," IEEE Transactions on Electromagnetic Compatibility, vol. 41, no. 4, pp. 307-319, 1999.

[12] M. Feliziani and F. Maradei, "Finite-difference time-domain modeling of thin shields," IEEE Transactions on Magnetics, vol. 36, no. 4, pp. 848-851, 2000.

[13] C. Buccella, M. Feliziani, F. Maradei, and G. Manzi, "Magnetic field computation in a physically large domain with thin metallic shields," IEEE Transactions on Magnetics, vol. 41, no, 5, pp. 1708-1711, 2005.

[14] M. Feliziani, "Subcell FDTD modeling of field penetration through lossy shields," IEEE Transactions on Electromagnetic Compatibility, vol. 54, no. 2, pp. 299-307, 2012.

[15] V. Nayyeri, M. Soleimani, and O. M. Ramahi, "A method to model thin conductive layers in the finite-difference timedomain method," IEEE Transactions on Electromagnetic Compatibility, vol. 56, no. 2, pp. 385-392, 2014.

[16] C. L. Holloway, M. Johansson, and M. S. Sarto, "An effective layer model for analyzing fiber composites," in Proceedings of the International Symposium on Electromagnetic Compatibility (EMC '98), pp. 511-516, Rome, Italy, September 1998.

[17] B. Gustavsen and A. Semlyen, "Rational approximation of frequency domain responses by vector fitting," IEEE Transactions on Power Delivery, vol. 14, no. 3, pp. 1052-1061, 1999.

[18] N. M. Nakhla, A. Dounavis, R. Achar, and M. S. Nakhla, "DEPACT: delay extraction-based passive compact transmission-line macromodeling algorithm," IEEE Transactions on Advanced Packaging, vol. 28, no. 1, pp. 13-23, 2005.

[19] A. Charest, M. S. Nakhla, R. Achar, D. Saraswat, N. Soveiko, and I. Erdin, "Time domain delay extraction-based macromodeling algorithm for long-delay networks," IEEE Transactions on Advanced Packaging, vol. 33, no. 1, pp. 219-235, 2010.

[20] Z.-Y. Huang, L.-H. Shi, B. Chen, and Y.-H. Zhou, "A new unconditionally stable scheme for FDTD Method using Associated Hermite orthogonal functions," IEEE Transactions on Antennas and Propagation, vol. 62, no. 9, pp. 4804-4809, 2014.

[21] S. Saboktakin and B. Kordi, "Time-domain distortion analysis of wideband electromagnetic-field sensors using Hermite-gauss orthogonal functions," IEEE Transactions on Electromagnetic Compatibility, vol. 54, no. 3, pp. 511-521, 2012.

[22] M. M. Rao, T. K. Sarkar, T. Anjali, and R. S. Adve, "Simultaneous extrapolation in time and frequency domains using hermite expansions," IEEE Transactions on Antennas and Propagation, vol. 47, no. 6, pp. 1108-1115, 1999. 
[23] M. M. Rao, T. K. Sarkar, R. S. Adve, T. Anjali, and J. F. Callejon, "Extrapolation of electromagnetic responses from conducting objects in time and frequency domains," IEEE Transactions on Microwave Theory and Techniques, vol. 47, no. 10, pp. 1964-1974, 1999.

[24] Y. Mengtao, A. De, T. K. Sarkar, J. Koh, and B. H. Jung, "Conditions for generation of stable and accurate hybrid TDFD MoM solutions," IEEE Transactions on Microwave Theory and Techniques, vol. 54, no. 6, pp. 2552-2563, 2006.

[25] Z. Bi, K. Wu, C. Wu, and J. Litva, "A dispersive boundary condition for microstrip component analysis using the FDTD method," IEEE Transactions on Microwave Theory and Techniques, vol. 40, no. 4, pp. 774-777, 1992.

[26] R. B. Schulz, V. C. Plantz, and D. R. Brush, "Shielding theory and practice," IEEE Transactions on Electromagnetic Compatibility, vol. 30, no. 3, pp. 187-201, 1988. 


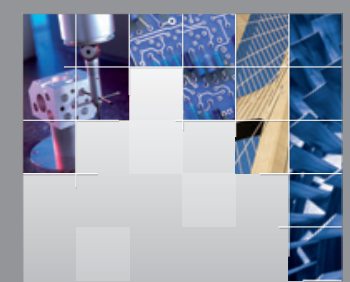

\section{Enfincering}
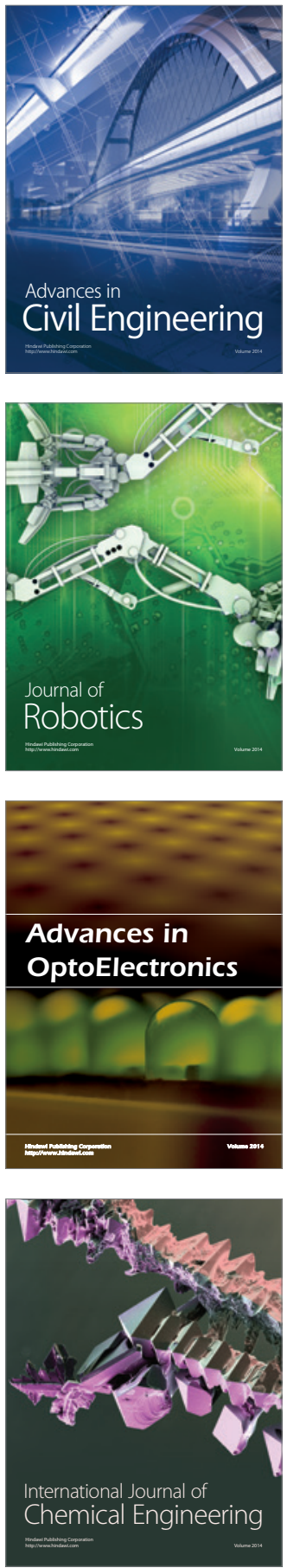

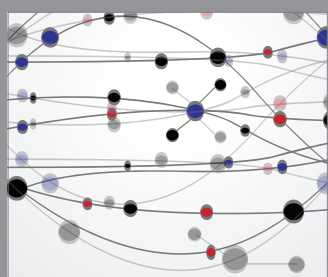

The Scientific World Journal

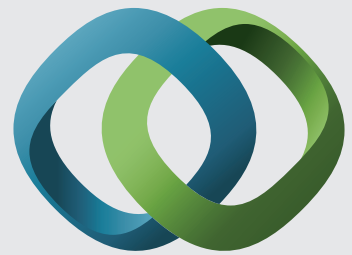

\section{Hindawi}

Submit your manuscripts at

http://www.hindawi.com
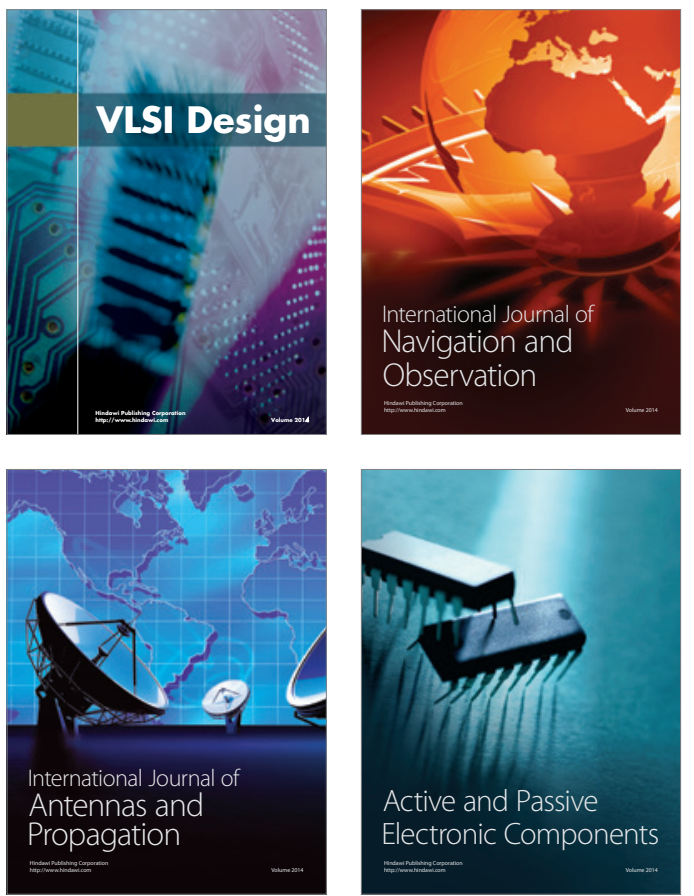
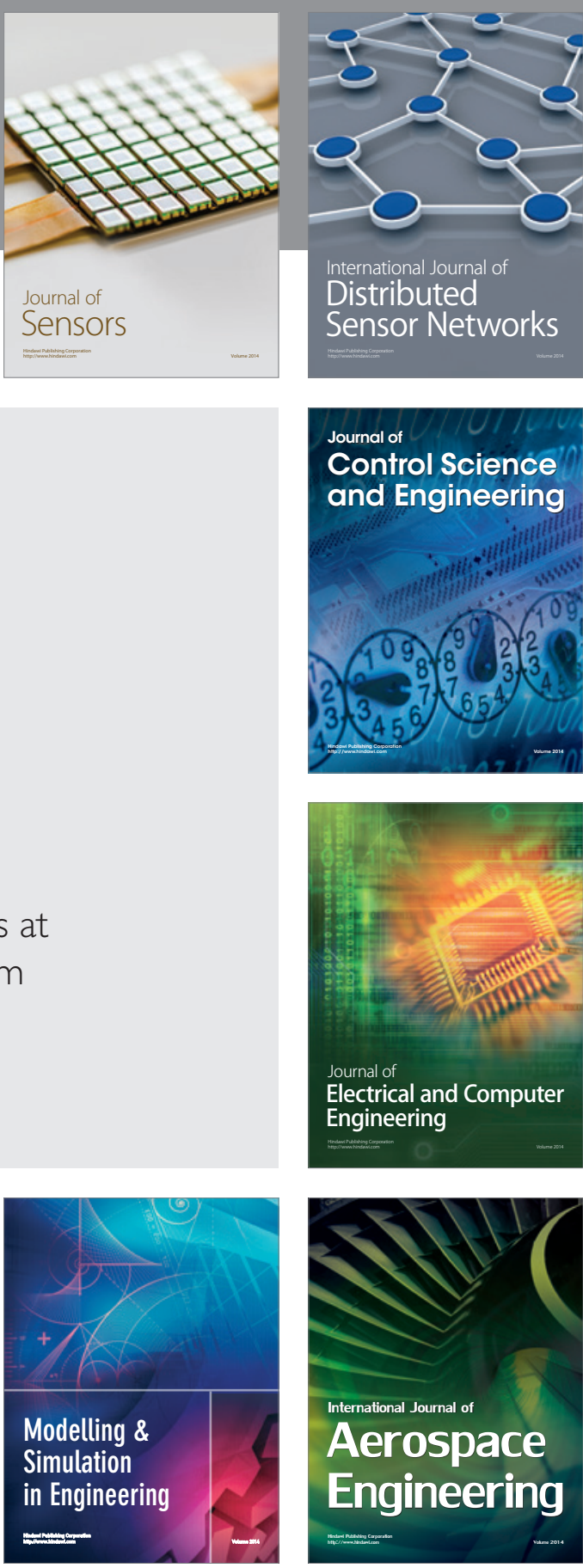

International Journal of

Distributed

Sensor Networks

Journal of

Control Science

and Engineering
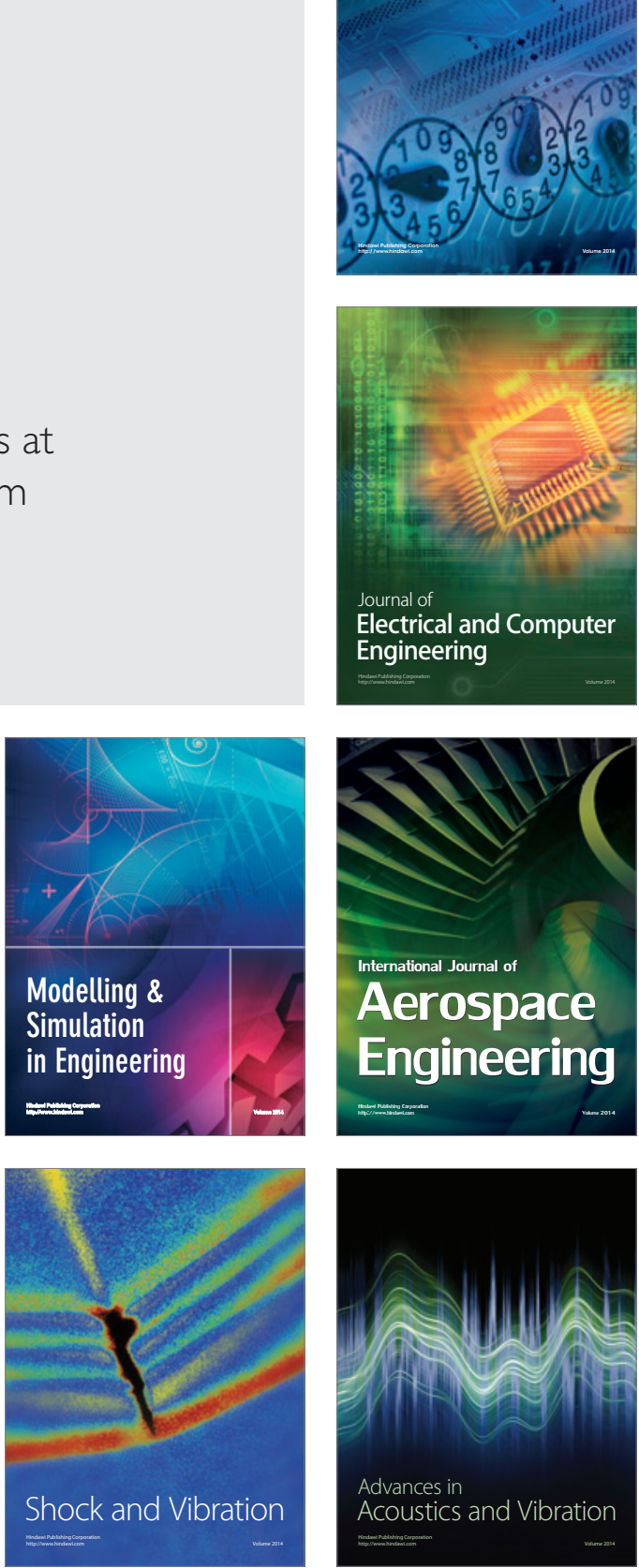